\title{
The protective effect of VSL\#3 on intestinal permeability in a rat model of alcoholic intestinal injury
}

Bing Chang ${ }^{1}$, Lixuan Sang ${ }^{2}$, Ying wang ${ }^{1}$, Jing Tong ${ }^{1}$, Dai Zhang ${ }^{1}$ and Bingyuan Wang ${ }^{{ }^{*}}$

\begin{abstract}
Background: This study aimed to investigate the mechanism of the probiotic VSL\#3 in acute alcoholic intestinal injury, and evaluate the effect of VSL\#3, glutamine,VSL\#3+glutamine and heat-killed VSL\#3 therapy in a rat model.

Methods: Six- to eight-week-old male wild-type rats were divided into seven groups. To establish the acute alcohol liver disease model, rats received three doses of corn starch dissolved in PBS/40\% alcohol administered intragastrically every 12 hours. Treatment groups received an intra-gastric dose of VSL\#3, Glutamine, heat-killed VSL\#3, or VSL\#3+Glutamine 30 minutes prior to alcohol administration. The placebo group was treated with PBS prior to alcohol administration. TNFa and endotoxin in plasma was measured by ELISA and Tachypleus Ameboctye Lysate assays, and electron microscopy, Western blotting, and reverse transcription polymerase chain reaction were used to identify the mechanisms of VSL\#3 in the regulation of epithelial permeability.
\end{abstract}

Results: First, compared with control group, endotoxin and TNFa in alcohol group was obviously high. At the same time, in VSL\#3 group,the expression of endotoxin and TNFa obviously lower than the alcohol group. And the trends of the expression of tight junction proteins in these groups were reversed with the change of endotoxin and TNFa. Second, compared the groups of VSL\#3 with glutamine,VSL\#3+glutamine and heat-killed VSL\#3, we found that both VSL\#3 and heat-killed VSL\#3, glutamine were as effective as VSL\#3+glutamine in the treatment of acute alcohol liver disease, the expression of endotoxin and TNFa were lower than the alcohol group, and tight junction proteins were higher than the alcohol group whereas the expression of tight junction proteins were higher in VSL\#3 + glutamine group than either agent alone, but have no significant difference.

Conclusion: We conclude that VSL\#3 treatment can regulate the ecological balance of the gut microflora, preventing passage of endotoxin and other bacterial products from the gut lumen into the portal circulation and down-regulating the expression of TNFa, which could otherwise down-regulate the expression of tight junction proteins and increase epithelial permeability.

Keywords: Intestinal barrier, TNFa, Tight junctions, VSL\#3, Glutamine

\section{Background}

Alcohol consumption is associated with the development of various medical disorders including alcoholic liver disease (ALD) and pancreatitis. Several studies have shown that the phenomenon of short-term excessive drinking is more common than addiction to alcohol, and that there is not always a dose-effect relationship between liver injury

\footnotetext{
* Correspondence: wby000216@163.com

'Department of Gastroenterology, First Affiliated Hospital of China Medical University, 110001 Shenyang, Liaoning Province, China

Full list of author information is available at the end of the article
}

and alcohol, environmental, and genetic factors [1,2]. There are currently no effective treatments for these diseases related with alcohol and, other than abstention, no preventive measures. The study presented here describes a potential new target for preventing alcoholic intestinal injury.

There is increasing evidence that the intestinal barrier plays a central role in the initiation of alcohol-induced tissue damage, and this role is most convincing for liver injury. Disruption of the intestinal barrier allows endotoxin and other bacterial products in the gut lumen to pass into the portal circulation and cause hepatic

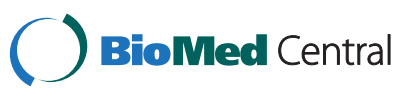


inflammation and the development of alcoholic steatohepatitis (ASH). This in turn can lead to alcoholic cirrhosis and liver failure, which is a causal factor in the development of alcoholic endotoxemia and hepatitis. Keshavarizian [3] found that intestinal barrier dysfunction was observed in alcoholics who also had liver disease. The intestinal barrier is formed by the epithelial cells and the tight junctions (TJs) between them [4], and provides barrier functions between luminal triggers and the host. Intestinal barrier dysfunction may lead to increased uptake of luminal antigens that promote mucosal inflammation.

The human gut harbors a large and dynamic bacterial community which plays a major role in human health. There are more than 1,000 species of microorganisms in the human intestine. The majority of these are anaerobic bacteria, such as clostridium, lactobacillus, Escherichia coli and bifidobacterium, and the total number has been estimated as more than $10^{14}$-ten times higher than the number of human cells $[5,6]$. Under normal circumstances, these maintain a balanced state and interact with the host, with profound effects on the host's ability to fight against infections. Previous studies have demonstrated that following damage to the liver there is reduced blood flow through the gut-liver axis, altered bile secretion, and increased epithelial permeability, leading to disruption of both the mucosal barrier and the ecological balance of the gut microflora [7-10]. Previous studies have also found that lactobacillus is significantly reduced, and enterobacter significantly increased [11].

Probiotics are non-pathogenic beneficial flora that act to regulate and maintain a stable intestinal environment and promote micro-ecological balance [12]. VSL\#3 is a probiotic mixture which has been frequently referred to in the literature, and contains live lyophilized Bifidobacterium breve, Bifidobacterium longum, Bifidobacterium infantis, Lactobacillus acidophilus, Lactobacillus plantarum, Lactobacillus paracasei, Lactobacillus bulgaricus and Streptococcus thermophilus.

In this study, we investigated the protective effect of VSL\#3 in alcoholic intestinal injury using an animal model. We found that VSL\#3 treatment can reduce colonic paracellular permeability and increase the expression of tight junction proteins (ZO-1 and occludin). Furthermore, the intestinal barrier prevents endotoxin and other bacterial products passing from the gut lumen into the portal circulation, and thereby protects against hepatic inflammation.

\section{Methods}

\section{Rats and treatments}

Six- to eight-week-old male WT rats (either littermates or age-matched, $200 \pm 10 \mathrm{~g}$ at the start of the experiment) were obtained from the Experimental Animal Center, China
Medical University. All rats in the study were used strictly in accordance with the National Institutions of Health Guide for the Care and Use of Laboratory Animals. This research was approved by the China Medical University Animals Committee (Approval Number: 2011-1538). They were divided into seven groups, and eight rats in each group.

Rats were deprived of food for 12 hours before induction of acute alcoholic liver injury. For the experiments, rats were divided into seven groups.

In the control group, eight rats were treated twice daily with corn starch which was dissolved in $200 \mu \mathrm{l}$ of PBS and administered via a gastric tube.

In the alcohol group, eight rats were given $40 \%$ alcohol ( $5 \mathrm{~g} / \mathrm{kg}$ body weight) through stomach feeding every 12 hours a total of three times.

In the VSL\#3, glutamine, heat-killed VSL\#3 and VSL\#3 + glutamine groups, rats were respectively given VSL\#3 (0.6 g/ $\mathrm{kg}$ body weight), glutamine $(0.3 \mathrm{~g} / \mathrm{kg}$ body weight), heat-killed VSL\#3 (0.6 g/kg body weight) or a combination of VSL\#3 and glutamine (VSL\#3 $0.6 \mathrm{~g} / \mathrm{kg}$ body weight, glutamine $0.3 \mathrm{~g} / \mathrm{kg}$ body weight) through stomach feeding 30 minutes prior to administration of alcohol as described above.

In the placebo group, rats were treated with PBS through stomach feeding before induction of alcohol liver injury.

The entire small intestine was collected, formalin fixed, and paraffin-embedded.

\section{Assessment of small intestinal tight junction proteins by electron microscopy}

A standard fixation procedure was used for conventional thin section electron microscopy. The procedure involved incubation with $\mathrm{OsO} 4$ alone ( 1 or $2 \%$ in phosphate buffer) at $0^{\circ} \mathrm{C}$ for $30 \mathrm{~min}$. After fixation, the small intestine was washed extensively in Veronal acetate buffer $(90 \mathrm{~mm}$, $\mathrm{pH}$ 6.0), stained by incubation at $0^{\circ} \mathrm{C}$ for $60 \mathrm{~min}$ in uranylmagnesium acetate $(0.5 \%)$ in the same buffer, washed again, dehydrated, and embedded. Thin sections were cut at $60 \mathrm{~nm}$ with a diamond knife and stained with uranyl acetate and lead citrate for viewing on a 200 CX transmission electron microscopeat $80 \mathrm{kV}$. Highmagnification pictures $(\times 10,000)$ were taken to evaluate the ultrastructure of small intestinal tight junctions.

\section{Measurement of TNF- $a$, endotoxin in plasma}

TNF- $\alpha$ levels in the media were assessed using a rat TNF- $\alpha$ ELISA according to the manufacturer's instructions. TNF- $\alpha$ concentrations were determined using a standard and values were normalized to total DNA present in the well using a DNA quantification kit.

Endotoxin was measured using the Tachypleus Amebocyte Lysate assay. Add $100 \mu \mathrm{l}$ of endotoxin test solution, endotoxin standard solution and samples into each 
hole of the micro plate and placed it tachypleus amebocyte lysate, $37^{\circ} \mathrm{C}$ for 10 minutes. And then add $100 \mu \mathrm{l}$ tachypleus amebocyte lysate into each of them, read the board at the wavelength of $340 \mathrm{~mm}$ once every 30 seconds.

\section{Western blot analysis}

Using snap-frozen small intestine specimens with histologically intact epithelium, we stripped the mucosa from the underlying submucosal tissue, homogenized and sonicated it, and transferred it into ice-cold lysis buffer with a protease inhibitor cocktail for $60 \mathrm{~min}$. Lysates were centrifuged and the protein content of the supernatant was determined using the BCA protein assay kit. Depending on the antibody used, equivalent protein concentrations of 10-75 $\mu \mathrm{g}$ were loaded in each lane of an SDS-polyacrylamide gel. Electrophoretically separated samples were transferred to an immobilon transfer membrane. Membranes were incubated with the respective primary antibodies and a corresponding peroxidase-conjugated secondary antibody. Blots were visualized by chemiluminescence using immobilon Western Chemiluminescent HRP substrate. After detection of specific tight junctions, all membranes were stripped with Restore Western Blot Stripping Buffer, and an immunoblot for $\beta$-actin was performed to ensure equal protein loading in each lane. Densitometry was performed for each protein detected in each group.

RNA isolation and reverse transcription-polymerase chain reaction

All of the plasmids were cloned using PCR. RNA extraction, reverse transcription polymerase chain reaction (RT-PCR), microarray. Primer sequences used in the RTPCR analyses are presented in supporting information. The following primer pairs were used for amplification:

occludin (sense, 5'-GCTATGAAACCGACTACACGAC A-3'; antisense, 5'-ACTCTCCAGCAACCAGCATCT-3'). ZO-1 (sense, 5'-AGGCTATTTCCAGCGTTTTGA-3'; antisense, 5'-AATCCTGGTGGTGGTACTTGC-3').

\section{Statistical analysis}

All data are expressed as mean $\pm \mathrm{SD}$ and were analyzed using one-way analysis of variance. $\mathrm{P}<0.05$ was considered statistically significant.

\section{Results and discussion Results}

Evaluation of tight junctions using electron microscopy

We studied tight junctions in the small intestine using electron microscopy to establish an index of loss of intestinal barrier integrity. Acute alcohol administration significantly disrupted the architecture of tight junctions of the small intestine. Supplementation with VSL\#3, glutamine or heat-killed VSL\#3 significantly protected the cytoarchitecture of the intestinal barrier, and VSL\#3 + Glutamine showed a more significant protective effect on tight junctions than the other treatment groups (Figure 1).

\section{Endotoxin and TNFa in plasma}

We assessed the expression of TNF $\alpha$ in plasma in the seven experimental groups using ELISA. Compared with the control group $(174.69 \pm 20.68)$, the expression of TNFa was higher in the alcohol group $(383.08 \pm 20.21)$. In the glutamine $(211.01 \pm 25.87)$, VSL\#3 (201.54 \pm 26.56), and heat-killed VSL\#3 $(197.85 \pm 17.97)$ groups the expression of TNF $\alpha$ was significantly lower than in the alcohol group, and there was no significant difference between these groups. The expression of TNF $\alpha$ in the VSL\#3 + glutamine group $(195.82 \pm 17.19)$ was lower than in the other three treatment groups, but this difference was not significant (Figure 2).

We assessed the level of plasma endotoxin in the seven experimental groups using the Tachypleus Amebocyte Lysate assay. Plasma endotoxin was higher in the alcohol group $(1.57 \pm 0.11)$ compared with the control group $(0.19 \pm 0.10)$. In the glutamine $(0.33 \pm 0.11)$, VSL $\# 3(0.34 \pm$ $0.10)$, and heat-killed VSL\#3 $(0.36 \pm 0.13)$ groups, plasma endotoxin was significantly lower than in the alcohol group, and there was no significant difference between these groups. The level of plasma endotoxin in the VSL $\# 3$ + glutamine group $(0.31 \pm 0.13)$ was lower than the other three treatment groups, but this was not significant (Figure 3).

\section{Tight junction mRNA and protein expression}

Tight junction mRNA and protein expression was analyzed in the small intestine of the seven groups by RTPCR and western blotting respectively. The trend of tight junction protein expression was the opposite of the change in endotoxin and TNFa. Specifically, in the alcohol group (occludin mRNA: $0.19 \pm 0.04$, occludin protein: $0.34 \pm$ 0.06; ZO-1 mRNA $0.19 \pm 0.05$, ZO-1 protein: $0.19 \pm 0.03$ ), expression of mRNA and protein for both occludin and ZO-1 was dramatically lower than the control group (occludin mRNA: $0.56 \pm 0.11$, occludin protein: $0.79 \pm$ 0.08; ZO-1 mRNA $0.95 \pm 0.10$, ZO-1 protein: $0.48 \pm 0.04$ ).

In the glutamine (occludin mRNA: $0.41 \pm 0.03$, occludin protein: $0.60 \pm 0.08$; ZO- 1 mRNA $0.66 \pm 0.08$, ZO- 1 protein: $0.34 \pm 0.05$ ), VSL $\# 3$ (occludin mRNA: $0.42 \pm 0.04$, occludin: $0.61 \pm 0.08$; ZO- 1 mRNA: $0.65 \pm 0.09$, ZO- 1 : $0.34 \pm 0.05$ ), and heat-killed VSL\#3 (occludin mRNA: $0.42 \pm 0.04$, occludin protein: $0.59 \pm 0.08$; ZO- 1 mRNA: $0.64 \pm 0.08, \mathrm{ZO}-1: 0.33 \pm 0.04)$ groups the expression of tight junction proteins was significantly higher than in alcohol group, and there was no significant difference between these groups. The expression of tight junction proteins in the VSL $\# 3$ + glutamine group (occludin mRNA: 


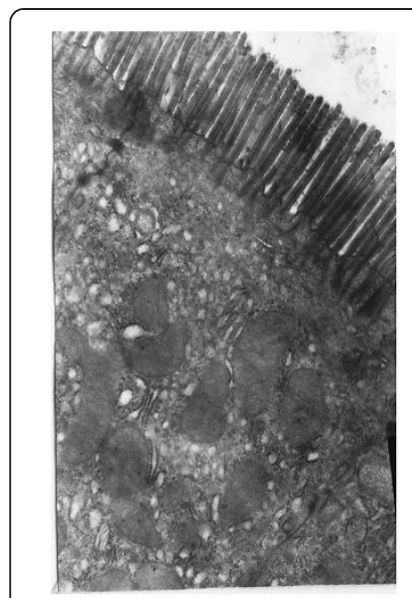

A

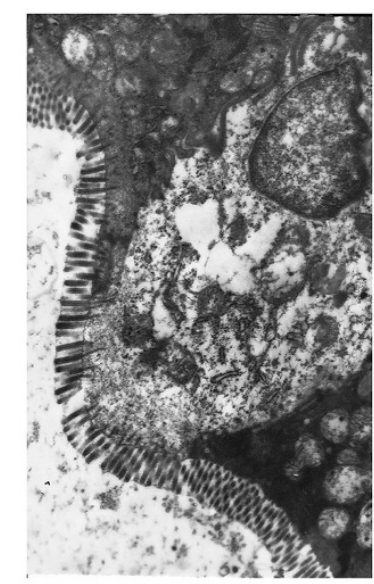

C

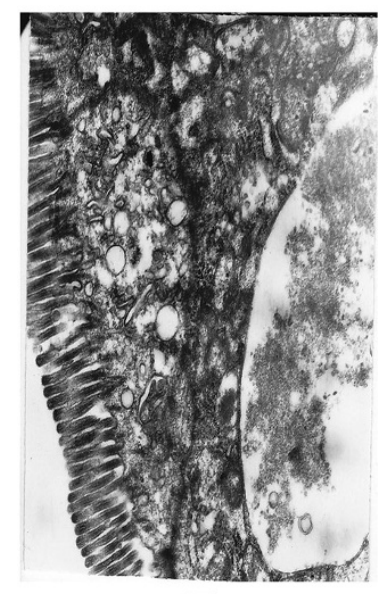

$\mathrm{E}$

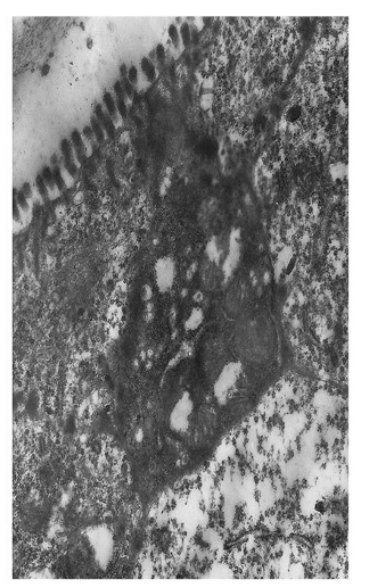

B

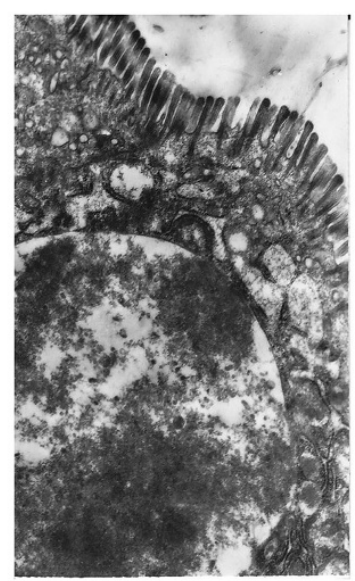

D

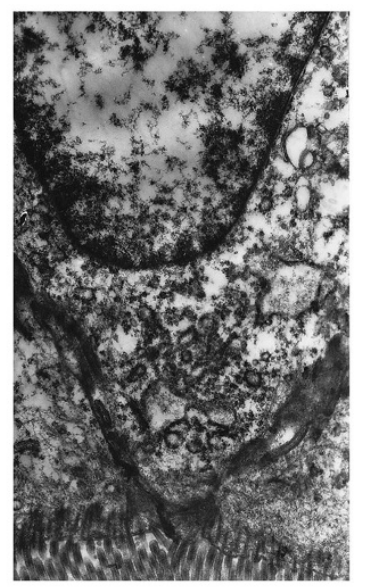

$\mathrm{F}$
Figure 1 The expression of tight junctions and microvilli of small intestine cell under electron microscopy. (A) The expression of tight junctions and microvili in control group; (B) The expression of tight junctions and microvili in alcohol group; (C) The expression of tight junctions and microvili in glutamine group; (D) The expression of tight junctions and microvili in VSL\#3 group; (E) The expression of tight junctions and microvili in VSL\#3+glutamine group; (F) The expression of tight junctions and microvili in placebo group.

$0.45 \pm 0.05$, occludin protein: $0.62 \pm 0.08$; ZO- 1 mRNA: $0.67 \pm 0.07$, ZO- 1 protein: $0.35 \pm 0.05$ ) was higher than the other three treatment groups, but this was not significant (Figures 4, 5, 6 and 7).

\section{Discussion}

Recently, the role of the gut microbiota in maintaining human health has attracted increasing attention. However, the relationship between gut microbiota and liver disease has not been extensively investigated. The growing understanding of the functional role of human gut microbiota is showing that this enormous microbial population is instrumental in the control of host energy [13-17]. The human gut microbiota is a complex bacterial community that is relatively stable over time [18-20]. Disruption of the microbiota can increase the risk of several health complications, including loss of colonization resistance against bacterial pathogens [21] and predisposition to autoimmune and allergic diseases [22]. Because the gut is a reservoir for microorganisms and contains more than 1,000 bacterial species, bacterial translocation from the gut due to dysbiosis of the gut microbiota and gut barrier failure may contribute to these infections [23,24]. Members of the Enterobacteriaceae family, Enterococcus spp. and the Bacteroides-Prevotella group are potentially pathogenic bacteria, while Lactobacillus, Bifidobacterium and F. prausnitzii are considered beneficial bacterial species for human well-being. Dysbiosis of the gut microbial ecosystem might be associated with the development of endotoxemia and eventually contribute to infections of liver, and TNF $\alpha$ is one of the most important mediators of inflammation.

Our previous study has shown that low grade intestinal inflammation induced by administering wild-type (WT) rats with alcohol results in liver injury. Impairment of the intestinal barrier function is associated with loss of tight junction proteins, including occludin and ZO-1. Tight junctions are the major determinants of paracellular permeability. Disruption of the intestinal barrier would allow endotoxin and other bacterial products in the gut lumen to pass into the portal circulation and thus potentially cause hepatic inflammation and the development of alcoholic steatohepatitis (ASH). This in turn would lead to alcoholic 


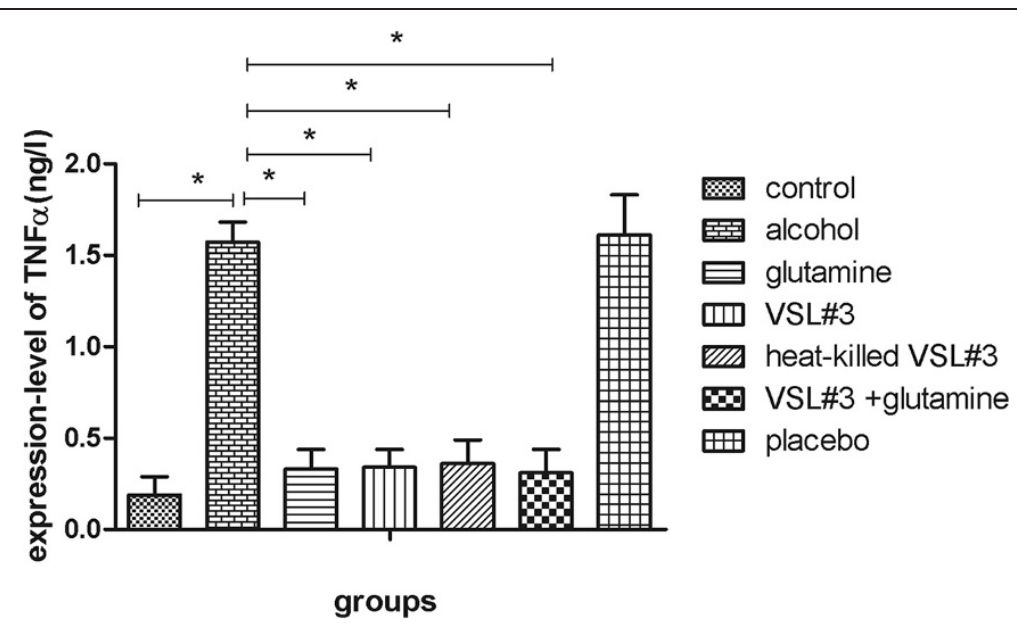

Figure 2 The expression of TNFa in each group measured by ELISA. ${ }^{*} P<0.05$ versus alcohol group.

cirrhosis and liver failure, which is a causal factor in the development of alcoholic endotoxemia and hepatitis.

Considering all of these possibilities, in the present study we used a rat model to investigate the effects of VSL\#3 administration, to explore its mechanism in the pathogenesis of acute alcohol liver disease, and to compare the effects of VSL\#3 with those of glutamine, a combination of VSL\#3 + glutamine, and heat-killed VSL\#3.

\section{The therapeutic mechanism of VSL\#3 in acute alcohol intestinal disease}

The present study was designed to investigate whether VSL\#3 could prevent liver injury by decreasing epithelial permeability. To achieve this we employed a model in which WT rats were fed with VSL\#3 before administration of alcohol. Our results demonstrate that expression of the tight junction proteins $\mathrm{ZO}-1$ and occludin was decreased in acute alcohol liver disease, and VSL\#3 treatment suppressed this effect by regulating the ecological balance of the gut microflora, preventing endotoxin and other bacterial products in the gut lumen from passing into the portal circulation and down-regulating the expression of TNF $\alpha$, which could otherwise down-regulate the expression of tight junction proteins and increase epithelial permeability, then endotoxin and other bacterial products pass from the gut lumen into the portal circulation, and lead to hepatic inflammation. Our results therefore suggest that probiotic-induced protection of epithelial

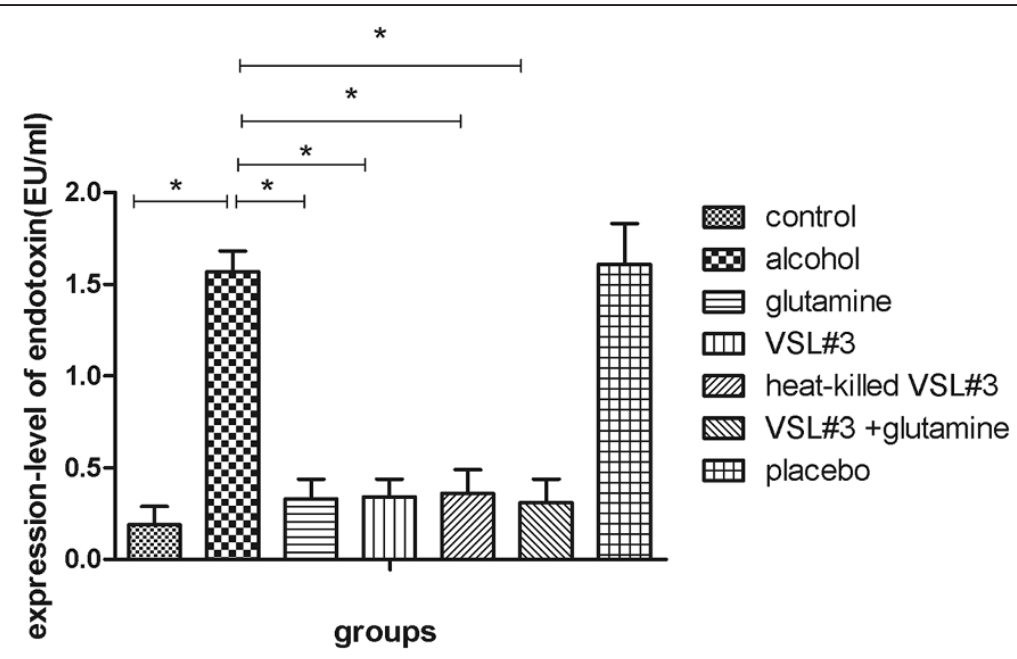

Figure 3 Levels of endotoxin in each group measured using the Tachypleus Amebocye Lysate assay. ${ }^{*} P<0.05$ versus alcohol group. 

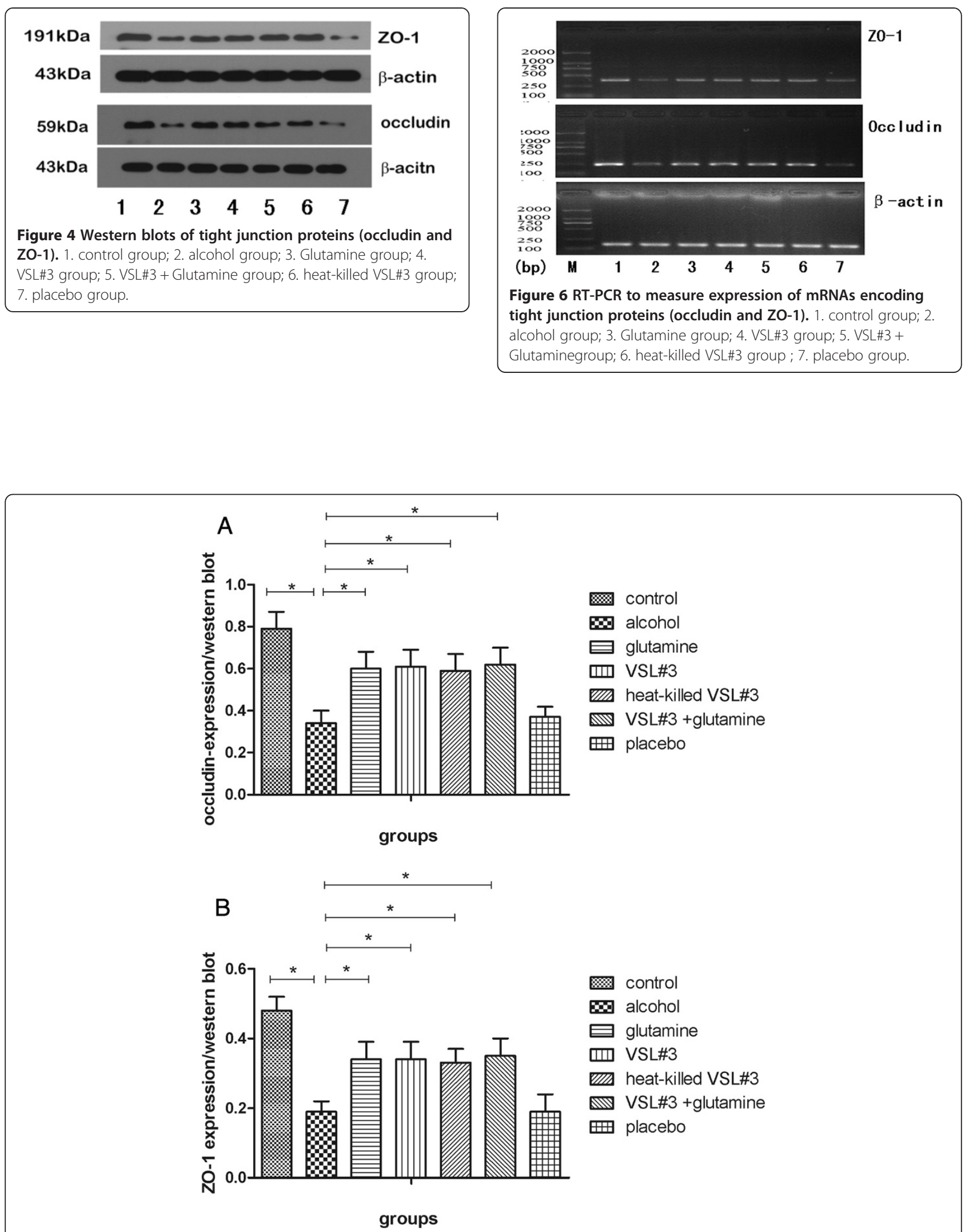

Figure 5 Western blot densitometry of tight junction protein (occludin and ZO-1) levels in each group. (A) Western blot densitometry of occludin; (B) Western blot densitometry of ZO-1. 

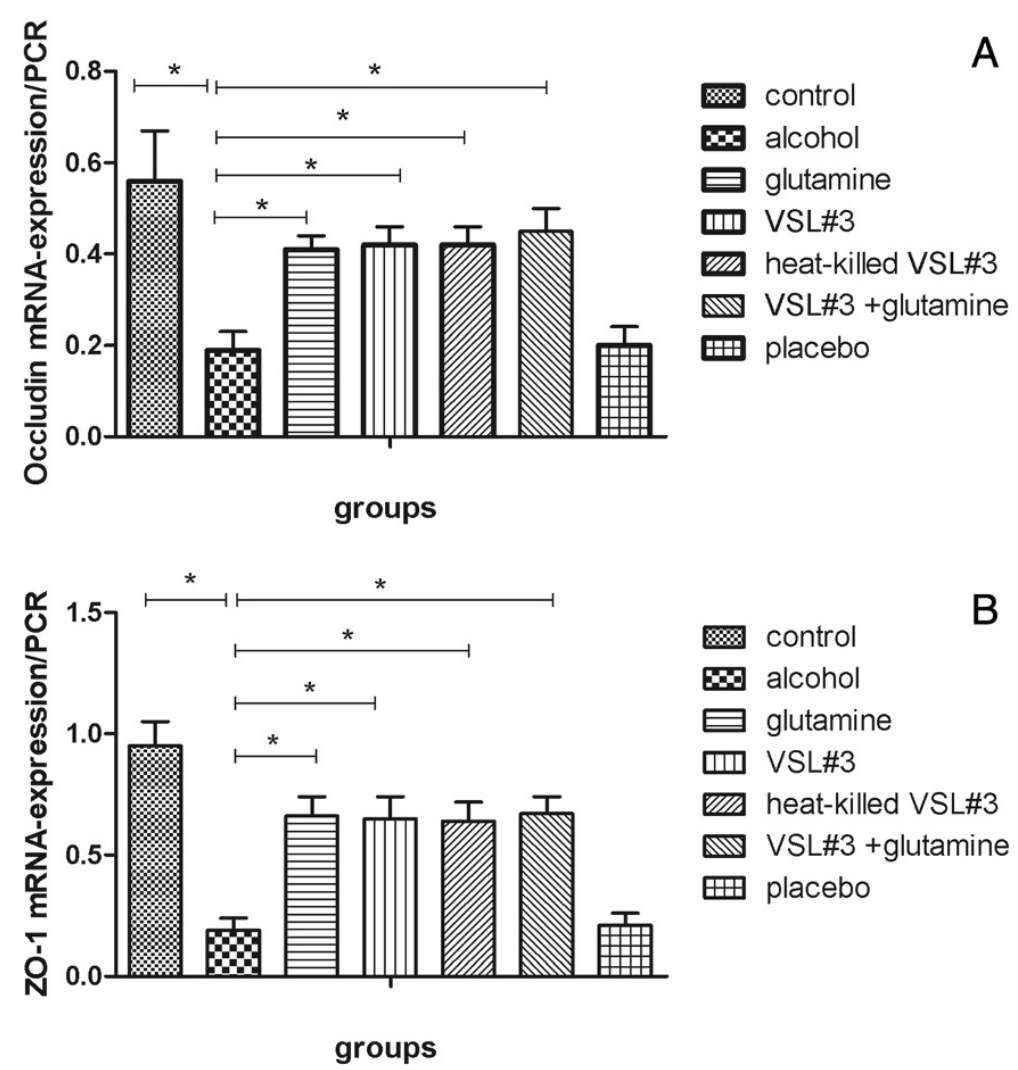

Figure 7 PCR densitometry of mRNA encoding tight junction proteins (occludin and ZO-1) in each group. (A) RT-PCR densitometry of occludin mRNA encoding; (B) RT-PCR densitometry of ZO-1 mRNA encoding.

barrier function is through prevention of changes in tight junction protein expression.

\section{Assessing the effect of VSL\#3, glutamine, VSL\#3 + glutamine and heat-killed VSL\#3}

Glutamine is a conditionally essential amino acid with immunomodulatory properties. It has a protective role in intestinal injury models, and may regulate proliferation of intestinal epithelial cells by modulating responsiveness to growth factors $[25,26]$. Small intestinal mucosa becomes atrophic when the gut is deprived of glutamine, for example during total parenteral nutrition [27]. Glutamine depletion can increase permeability of the gut which promotes translocation of luminal bacteria and toxins [28]. Glutamine has been shown to protect intestinal epithelial cells during physiological stress because it is required for stress-induced heat shock protein expression $[29,30]$ such as in experimental enterocolitis [31,32]. Glutamine most likely protects the gut via mucosal healing and a decrease in bacterial translocation [33]. Moreover, some studies have reported that glutamine down-regulated the intestinal inflammatory response in experimental models [31,34-36] by modulating the nuclear factor-kB (NF-kB) pathway [37-40].
The aim of the study was therefore to assess the effects of glutamine and VSL\#3, either alone or in combination, on acute alcohol liver disease, and to compare the effects of VSL\#3 and heat-killed VSL\#3. We found that both VSL\#3 and heat-killed VSL\#3 were as effective as glutamine in the treatment of acute alcohol liver disease, whereas the combination of VSL\#3 and glutamine therapy efficacy was more effective than either agent alone (although it showed no significant difference compared with the other groups).

\section{Conclusions}

The probiotic mixture VSL\#3 and heat-killed VSL\#3 were as effective as the traditional agent glutamine in rats with experimental acute alcohol liver disease, whereas the combination of VSL\#3 and glutamine was more effective than either alone. All of these treatments can prevent endotoxin and other bacterial products in the gut lumen from passing into the portal circulation, decrease the production of TNF $\alpha$ and increase the expression of tight junction proteins, thus reducing paracellular intestinal permeability. 


\section{Competing interest}

The authors declare that they have no competing interests.

\section{Authors' contributions}

BC and LS conceived and designed the study. BC and YW did the experiment technology. BC, JT, and BW did statistical analyses and interpreted results. All drafts of the reports, including the final version, were written by BC and LS and revised by BW. All authors read and approved the final paper.

\section{Acknowledgments}

We would like to thank Dr Cheng for supply and care of the animals. We also extend our grateful thanks to Dr Li for his help with the English text.

\section{Author details}

'Department of Gastroenterology, First Affiliated Hospital of China Medical University, 110001 Shenyang, Liaoning Province, China. ${ }^{2}$ Department of Cadre Ward II, First Affiliated Hospital of China Medical University, 110001 Shenyang, Liaoning Province, China.

Received: 30 May 2013 Accepted: 13 October 2013

Published: 20 October 2013

\section{References}

1. Stewart S, Jones D, Day CP: Alcoholic liver disease: new insights into mechanisms and preventative strategies. Trends Mol Med 2001, 7(9):408-413.

2. Rouault TA: Hepatic iron overload in alcoholic liver disease: why does it occur and what is its role in pathogenesis? Alcohol 2003, 30(2):103-106.

3. Keshavarzian A, Holmes EW, Patel M, et al: Leaky gut in alcoholic cirrhosis: a possible mechanism for alcohol-induced liver damage. Am J Gastroenterol 1999, 94(1):200-207.

4. Ma TY, Iwamoto GK, Hoa NT, et al: TNF-alpha-induced increase in intestinal epithelial tight junction permeability requires NF-kB activation. Am J Physiol Gastrointest Liver Physiol 2004, 286(3):G367-G376.

5. Bengmark S: Ecological control of the gastroinstinal tract: the role of probiotic flore. Gut 1998, 42(1):2-7.

6. Simon GL, Gorbach SL: Intestinal flora in health and disease. Gastroenterology 1984, 86(1):174-193.

7. Guarner C, Runyon BA, Young S, et al: Intestinal bacterial overgrowth and bacterial translocation in cirrhotic rats with ascites. J Hepatol 1997 26(6):1372-1378

8. MacFie J, O'Boyle C, Mitchell C, et al: Gut origin of sepsis: a prospective study investigating associations between bacterial translocation, gastric microflora, and septic morbidity. Gut 1999, 45(2):223-228.

9. Othman M, Agfiero R, Lin HC: Alterations in intestinal microbial flora and human disease. Curr Opin Gastroenterol 2008, 24(1):11-16.

10. Shimizu K, Ogura H, Goto M, et al: Altered gut flora and environment in patients with severe SIRS. J Trauma 2006, 60(1):126-133.

11. Li LJ, Wu ZW, Xiao DS, et al: Changes of gut flora and endotoxin in rats with D-galactosamine induced acute liver failure. World J Gastroenterol 2004, 10(14):2087-2090.

12. Fuller R: Probiotics in man and animals. J Appl Bacterio/ 1989, 66(5):365-378.

13. Abubucker S, Segata N, Goll J, et al: Metabolic reconstruction for metagen -omic data and its application to the human microbiome. PLoS Comput Biol 2012, 8(6):e1002358.

14. Brown CT, Davis-Richardson AG, Giongo A, et al: Gut microbiome meta genomics analysis suggests a functional model for the development of autoimmunity for type 1 diabetes. PLoS One 2011, 6(10):e25792.

15. Lakhdari $O$, Cultrone A, Tap J, et al: Functional metagenomics: a high through- put screening method to decipher microbiota-driven NF-KB modulation in the human gut. PLOS One 2010, 5(9):e13092.

16. Murphy EF, Cotter PD, Healy S, et al: Composition and energy harvesting capacity of the gut microbiota: relationship to diet, obesity and time in mouse models. Gut 2010, 59(12):1635-1642.

17. Qin J, Li R, Raes J, et al: A human gut microbial gene catalogue established by metagenomic sequencing. Nature 2010, 464(7285):59-65.

18. Claesson MJ, Cusack S, O'Sullivan O, et al: Composition, variability, and temporal stability of the intestinal microbiota of the elderly. Proc Natl Acad Sci USA 2011, 108(Suppl 1):4586-4591.
19. Jalanka-Tuovinen J, Salonen A, Nikkila J, et al: Intestinal microbiota in healthy adults: temporal analysis reveals individual and common core and relation to intestinal symptoms. PLoS One 2011, 6(7):e23035.

20. Nam YD, Jung MJ, Roh SW, et al: Comparative analysis of Korean human gut microbiota by barcoded pyrosequencing. PLoS One 2011, 6(7):e22109.

21. Reeves $A E$, Theriot $C M$, Bergin IL, et al: The interplay between microbiome dynamics and pathogen dynamics in a murine model of Clostridium difficile Infection. Gut Microbes 2011, 2(3):145-158.

22. Sjogren YM, Jenmalm MC, Bottcher MF, et al: Altered early infant gut micro- biota in children developing allergy up to 5 years of age. Clin Exp Allergy 2009, 39(4):518-526

23. Deitch EA: Bacterial translocation or lymphatic drainage of toxic products from the gut: what is important in human beings? Surgery 2002, 131(3):241-244.

24. Rajilić-Stojanović M, Smidt H, de Vos WM: Diversity of the human gastrointestinal tract microbiota revisited. Environ Microbiol 2007, 9(9):2125-2136.

25. Ko TC, Beauchamp RD, Townsend CM Jr, et al: Glutamine is essential for epidermal growth factor-stimulated intestinal cell proliferation. Surgery 1993, 114(2):147-153.

26. Scheppach W, Loges C, Bartram P, et al: Effect of free glutamine and alanyl-glutamine dipeptide on mucosal proliferation of the human ileum and colon. Gastroenterology 1994, 107(2):429-434.

27. Buchman AL, Moukarzel AA, Bhuta S, et al: Parenteral nutrition is associated with intestinal morphologic and functional changes in humans. JPEN J Parenter Enteral Nutr 1995, 19(6):453-460.

28. Van der Hulst RR, van Kreel BK, von Meyenfeldt MF, et al: Glutamine and the preservation of gut integrity. Lancet 1993, 341(8857):1363-1365.

29. Musch MW, Ciancio MJ, Sarge K, et al: Induction of heat shock protein 70 protects intestinal epithelial IEC-18 cells from oxidant and thermal injury. Am J Physiol 1996, 270(2 pt 1):C429-C436.

30. Wischmeyer PE, Musch MW, Madonna MB, et al: Glutamine protects intestinal epithelial cells: role of inducible HSP70. Am J Physiol 1997 272(2 pt 1):G879-G884

31. Ameho CK, Adjei AA, Harrison EK, et al: Prophylactic effect of dietary glutamine supplementation on interleukin 8 and tumour necrosis factor alpha production in trinitrobenzene sulphonic acid induced colitis. Gut 1997, 41(4):487-93.

32. Vicario $M$, Amat $C$, Rivero $M$, Moreto $M$, et al: Dietary glutamine affects mucosal functions in rats with mild DSS-induced colitis. J Nutr 2007 137(8):1931-1937.

33. Ziegler TR, Evans ME, Fernandez-Estivariz C, et al: Trophic and cytoprotective nutrition for intestinal adaptation, mucosal repair, and barrier function. Annu Rev Nutr 2003, 23:229-61.

34. Lecleire S, Hassan A, Marion-Letellier R, et al: Combined glutamine and argin- ine decrease proinflammatory cytokine production by biopsies from Crohn's patients in association with changes in nuclear factor-kappa B and p38 mitogen-activated protein kinase pathways. J Nutr 2008, 138(12):2481-2486.

35. Coeffier M, Marion R, Ducrotte $P$, et al: Modulating effect of glutamine on IL1beta-induced cytokine production by human gut. Clin Nutr 2003, 22(4):407-413.

36. Huang Y, Li N, Liboni K, et al: Glutamine decreases lipopolysaccharideinduced IL-8 production in Caco-2 cells through a non-NF-kappaB p50 mechanism. Cytokine 2003, 22(2-3):77-83.

37. Liboni KC, Li N, Scumpia PO, et al: Glutamine modulates LPS-induced IL-8 production through IkKB/NF-KB in human fetal and adult intestinal epithelium. J Nutr 2005, 135(2):245-251.

38. Hubert-Buron A, Leblond J, Jacquot A, et al: Glutamine pretreatment reduces IL-8 production in human intestinal epithelial cells by limiting IKBa ubiquitination. J Nutr 2006, 136(6):1461-1465.

39. Fillmann H, Kretzmann NA, San-Miguel B, et al: Glutamine inhibits over-expression of pro-inflammatory genes and down-regulates the nuclear factor kappaB pathway in an experimental model of colitis in the rat. Toxicology 2007, 236(3):217-226.

40. Brasse-Lagnel C, Lavoinne A, Loeber D, et al: Glutamine and interleukin-1 beta interact at the level of Sp1 and nuclear factor-kappaB to regulate argininosuccinate synthetase gene expression. FEBS J 2007, 274(20):5250-5262.

doi:10.1186/1471-230X-13-151

Cite this article as: Chang et al:: The protective effect of VSL\#3 on intestinal permeability in a rat model of alcoholic intestinal injury. BMC Gastroenterology 2013 13:151. 